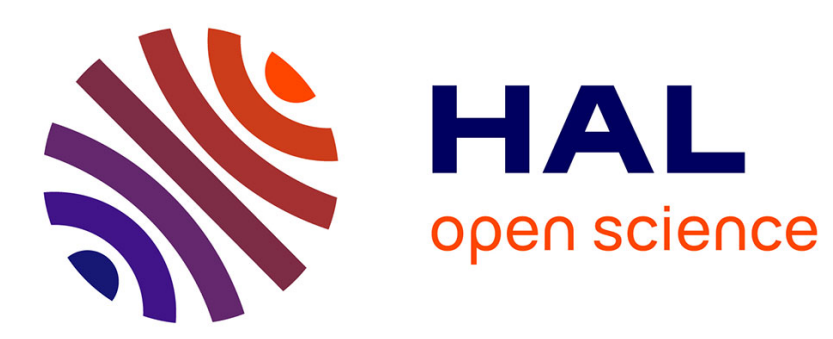

\title{
Phonon modes and stability of GaS up to 200 kilobars
}

Alain Polian, J. Chervin, J. Besson

\section{To cite this version:}

Alain Polian, J. Chervin, J. Besson. Phonon modes and stability of GaS up to 200 kilobars. Physical Review B: Condensed Matter (1978-1997), 1980, 22 (6), pp.3049 - 3058. 10.1103/PhysRevB.22.3049 . hal-01820703

\section{HAL Id: hal-01820703 https://hal.science/hal-01820703}

Submitted on 22 Jun 2018

HAL is a multi-disciplinary open access archive for the deposit and dissemination of scientific research documents, whether they are published or not. The documents may come from teaching and research institutions in France or abroad, or from public or private research centers.
L'archive ouverte pluridisciplinaire HAL, est destinée au dépôt et à la diffusion de documents scientifiques de niveau recherche, publiés ou non, émanant des établissements d'enseignement et de recherche français ou étrangers, des laboratoires publics ou privés. 


\title{
Phonon modes and stability of GaS up to 200 kilobars
}

\author{
A. Polian, J. C. Chervin, and J. M. Besson \\ Laboratoire de Physique des Solides, Associé au Centre National de la Recherche Scientifique, Université Pierre et Marie Curie, 4, Place \\ Jussieu, 75230 Paris Cédex 05, France \\ (Received 31 March 1980)
}

\begin{abstract}
Raman scattering and absorption measurements of $\mathrm{GaS}$ under high pressure are reported. The pressure coefficients of one-phonon modes, as well as 2-LA and 2-TA combinations, have been measured. The latter is negative. The anomalous pressure coefficient of the intralayer $E_{1 g}$ vibration is discussed in terms of its shear character. Accidental energetic degeneracy, at $\simeq 100 \mathrm{kbars}$, of an $A_{1 \mathrm{~g}}$ mode with a $2-\mathrm{LA}$ density of states broadens the Raman line. Other line broadenings which are induced by pressure are discussed. This behavior is shown to be an intrinsic property of the crystal and not inhomogeneous strain broadening, since the original method we use minimizes stress gradients even above $100 \mathrm{kbars}$ in the diamond anvil cell. A structural phase transition above $190 \mathrm{kbars}$ is reported. The new phase behaves like a three-dimensionally bonded network.
\end{abstract}

\section{INTRODUCTION}

High-pressure studies on phonons have taken a new dimension with the advent of the diamond anvil cell techniques, especially by Raman scattering measurements, which are readily accessible in this type of apparatus. The extended range of pressures (well above $100 \mathrm{kbars}$ ) which it provides gives a powerful tool for shifting phonon frequencies and identifying the nature of the corresponding restoring forces. In the case of molecular, fibrous, or layer crystals, the variation of weak forces binding molecules, fibers, or layers may change by an order of magnitude, as is shown here, for instance. Moreover, the differences in pressure coefficients lead to the possibility of crossover, ${ }^{1}$ or splitting, ${ }^{2}$ of individual frequencies. Interference effects can then be used to identify density-of-states singularities in the range of pressure, however, where the relevant physical quantity, that is, the natural width, can be isolated from spurious (inhomogeneous-stress) broadening. Comparatively few studies have been published on the pressure variation of layer crystals, or indeed low-dimensionality solids. ${ }^{2-6}$ In this paper we study one of the most classical members of the gallium-indium chalcogenides family GaS, which stands out for its stability since it has only one polytype, and for the simplicity of its phonon spectrum, due to its high symmetry.

We have used a new method of pressurization which gives homogeneous-stress conditions above 200 kbars. ${ }^{7}$ Apart from the measurements of the shift with pressure of the Raman-active one-phonon lines which essentially confirm lower-pressure data,,$^{5,6}$ we could also measure for the first time, the pressure coefficients of the edge-of-the-zone LA and TA vibrations, the latter being negative, as in three-dimensional semiconductors. The low value of the pressure coefficient of the $E_{1 g}$ vibra- tion (intralayer shear or rigid half-layer shear) is explained on the basis of its displacement pattern which makes it analogous to edge-of-the-zone TA vibration $s^{8}$ as regards the destabilizing effect of pressure upon electronic contributions to restoring forces.

The method of pressurization which was used ${ }^{7,9,10}$ provides better than $1 \%$-pressure homogeneity: It was thus possible to observe the natural full width at half maximum (FWHM) of several phonons and identify several interferences with densities of states away from $\Gamma$. Finally, a solid-solid phase transition above $190 \mathrm{kbars}$ has been identified. It represents the only other known crystalline modification of this compound and its characteristics strongly suggest collapse of interlayer distances, increase of interlayer forces, and at least a step towards three-dimensional binding.

\section{EXPERIMENTAL}

GaS samples are taken from vapor-grown hexagonal needles about $100 \mu \mathrm{m}$ in diameter. Those crystals have the usual $\beta$ structure $\left(D_{6 h}^{4}\right.$ space group) and the $c$ axis is parallel to the length of the needles. Slabs 10 to $30 \mu \mathrm{m}$ in thickness are cleaved with a razor blade, perpendicular to the $c$ axis.

The diamond anvil cell ${ }^{7}$ is used with a $150-\mu \mathrm{m}$ Inconel 750 gasket. The gasket hole, $300 \mu \mathrm{m}$ at the start, is reduced to about $15 \mu \mathrm{m}$ in diameter by prestraining between the diamonds, the cell being empty. This procedure permits us to reach regularly pressures up to $300 \mathrm{kbars}$. The pressurizing $^{11}$ fluid is the classical methanol-ethanol mixture and ruby chips, typically $30 \mu \mathrm{m}$ in diameter, are placed in the cell, together with the GaS samples.

In Raman experiments, the 530.2- and the 647.1$\mathrm{nm}$ lines of a $\mathrm{Kr}^{+}$laser are used, the incident 
power on the sample being kept below $100 \mathrm{~mW}$. Optical-transmission experiments are done with a Leitz metallographic microscope with a quartz-iodine lamp as a source. In both sets of experiments a Coderg T 800 triple monochrometer has been used.

Since some of the results presented here depend upon the stress homogeneity within the cell, the experimental procedure deserves some discussion: The precision given by the shift of the ruby lines as a pressure gauge is limited by the stress gradients within the cell in regions above $100 \mathrm{kbars}$, where the ethanol-methanol mixture becomes a very rigid glass. ${ }^{12}$ There is then no way to get, strictly speaking, hydrostatic conditions. Nevertheless, it has been shown that homogeneous stress can be obtained ${ }^{7}$ far above 100 kbars by rapid pressurizing of the experimental volume.

Inhomogeneous conditions in the cell lead to axial strains and differences in the hydrostatic component of the stress, at different points of the samples. This results in:

asymmetric broadening of the $R_{1}$ and $R_{2}$ lines of the ruby;

increase in the spectral distance of the $R_{1}-R_{2}$ maxima.

On the contrary, under hydrostatic or at least uniform conditions, ${ }^{7,11}$ both the $R_{1}$-peak linewidth and the $R_{1}-R_{2}$ spectral distance slightly decrease.

Clearly, stress inhomogeneity, as measured by the emission lines of a single ruby sample, will depend on the size of the sample, so that this can be only a qualitative measurement. Nevertheless, the sensibility of the ruby line shape to stress gradients is such that the onset of moderate inhomogeneity ( $1 \mathrm{kbar}$ ) is easily detected, as shown in Ref. 7, where it was shown that a rapid increase of pressure could overcome the kinetics of the liquid to glass transition, and freeze in uniform stress to at least $200 \mathrm{kbars}$, provided the sample volume stay centered throughout the process. This method was improved upon here by noting that, after pressurizing rapidly (a few minutes) up to the highest required pressure, slow depressurization (over a few hours) would still maintain homogeneous-stress conditions within experimental precision ( $1 \%$ in our experiments).

This is shown in Fig. 1, where the $R_{1}$ luminescence peak of the ruby chips has been normalized to the same height and same position to allow comparison at different pressures:

Curve 1 (upper abscissa scale) is at room pressure before and after the experiment.

Curve 2 (middle scale) is after pressurization to $235 \mathrm{kbars}$ in one minute. The emission spectrum does not evolve for hours afterwards, thus indicating stable and uniform stress conditions.

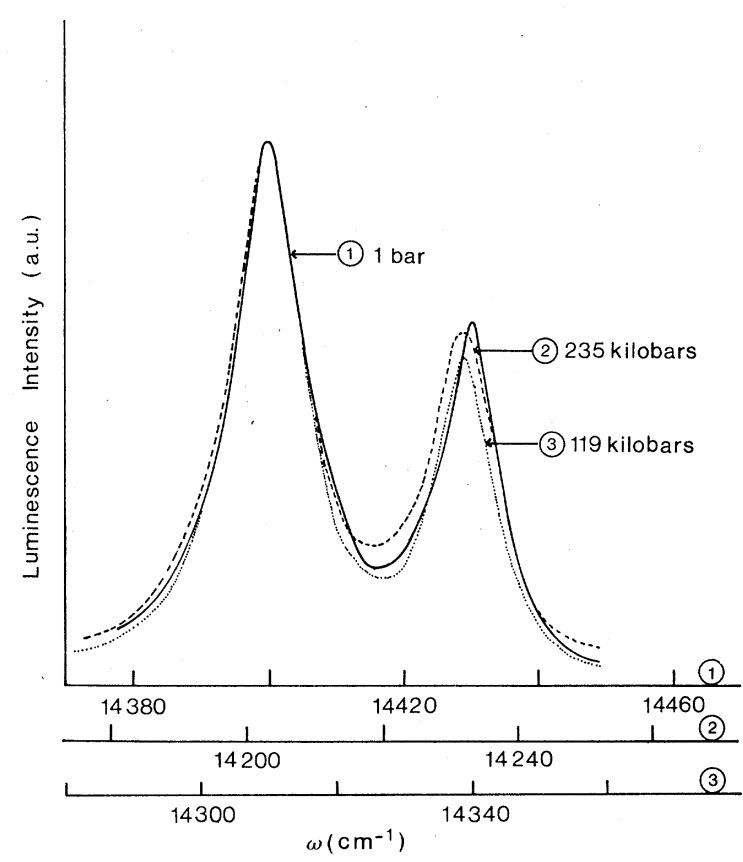

FIG. 1. Ruby luminescence spectrum. Full line: curve 1 , room pressure. Dashed line: curve 2, highest recorded pressure 235 kbars. Dotted line: curve 3, $119 \mathrm{kbar}$-decreasing from the previous pressure.

Curve 3 (lower scale) was taken after several successive depressurization steps from 235 to 119 kbars, the whole process lasting over one day.

Examination of those data shows that (i) the broadening of the $R_{1}$ peak (FWHIM) is less than 0.5 $\mathrm{cm}^{-1}$, which corresponds to less than $1 \mathrm{kbar}$ (1 kbar $\sim 0.753 \mathrm{~cm}^{-1}$ ) (ii) At 235 kbars the $R_{2}$ line is broadened by $1.5 \mathrm{~cm}^{-1}$ corresponding to 2 -kbar inhomogeneity. At $119 \mathrm{kbars}$, on the contrary, there is no measurable broadening (iii) The $R_{1}-R_{2}$ spectral distance is decreased by $1.2 \mathrm{~cm}^{-1}$ between 0 and $235 \mathrm{kbars}$. This decrease of $0.5 \mathrm{~cm}^{-1} / 100$ kbars is similar to that found under hydrostatic $\mathrm{c}^{11}$ or uniform ${ }^{7}$ conditions and shows immeasurable inhomogeneity. Thus, using the most pessimistic estimate (ii), the pressure gradients obtained by this method are at most $1 \%$ of the nominal pressure.

The results described thereafter were obtained with this procedure. It should be noted that the same behavior was observed ${ }^{10}$ at higher pressures (250 kbars) and that this method should provide uniform stress conditions far above this value.

\section{RESULTS}

A. Optical behavior under pressure

The gap of $\mathrm{GaS}$ is at $2.55 \mathrm{eV}$ at $300 \mathrm{~K}$ and its pressure coefficient is $-11 \times 10^{-6} \mathrm{eV} /$ bar at room pressure. ${ }^{6,13}$ The nonlinearity of the variation of 
the gap with pressure has been noted before ${ }^{6}$ and, at $180 \mathrm{kbars}$, the gap is still in the vicinity of 1.80 eV. At this pressure, GaS suddenly changes from dark red to black. The crystal remains opaque above this pressure and remains so down to 100 kbar. For that reason, only the two most intense Raman modes could be observed in this pressure region, that is, the $A_{1 g}$ vibrations, at $188 \mathrm{~cm}^{-1}$ and $360 \mathrm{~cm}^{-1}$ under ordinary conditions. When pressure is decreased from 100 to $30 \mathrm{kbars}$, the absorption edge shifts again to higher energies but remains several hundreds of meV lower than during the initial increase of pressure. Figure 2 shows the absorption edges at 30 kbars upon increase of pressure (curve 1) and after pressurization to over 200 kbars (curve 2). Further decrease of pressure below $30 \mathrm{kbars}$ brings about crumbling of the sample to microcrystals.

\section{B. Pressure dependence of phonon frequencies}

The variation in frequency of the Raman-active modes is shown in Fig. 3. Actually, the $E_{1 g}$ modes are not symmetry allowed in the backscattering $Z(X X) \bar{Z}$ geometry that was used here. They are activated in our crystals, by a high density of defects, presumably due here to the screw dislocations along the needle axis. This is a most general behavior in this family of crystals. ${ }^{14}$ Full dots and circles represent the one- and two-phonon modes, respectively. In the high-pressure modification, above $180 \mathrm{kbars}$, only the $A_{1 g}$ modes were observable, being stronger than other features in the spectrum. No distinct discontinuity was apparent after the phase transition for those intralayer modes. The only new feature which we could observe in the high-pressure modification of $\mathrm{GaS}$ is a mode around $60 \mathrm{~cm}^{-1}$ (stars in Fig. 3) which was followed from $120 \mathrm{kbars}$ down. The table displays

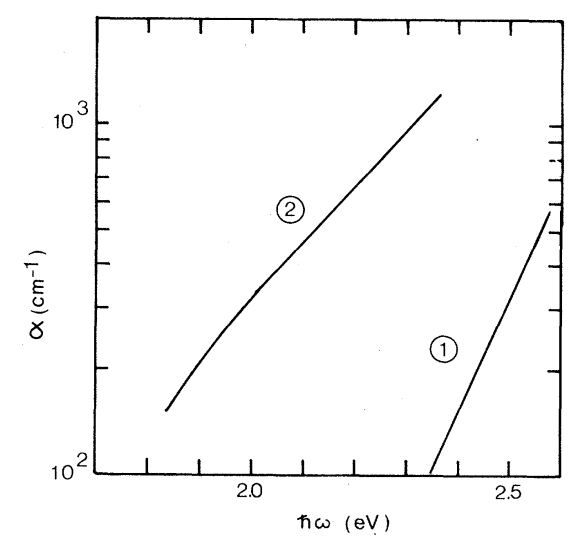

FIG. 2. Absorption coefficient at $30 \mathrm{kbars}$, at increasing pressure (1) and decreasing from 235 kbars (2). the relative pressure coefficients $(1 / \nu)(d \nu / d P)$ of the one- and two-phonon modes at zero pressure. In order to compare with other crystals (next section) we computed an isotropic Grüneisen parameter using the values of isothermal compressibility given for GaSe, since this quantity has not been measured in GaS. The volume compressibility was taken to be $\chi=(1 / \nu)(d \nu / d P)=2 \chi_{\perp}+\chi_{\|}=1.3 \times 10^{-6}$ bar $^{-1}$. Compressibilities perpendicular $\left(\chi_{\perp}\right)$ and parallel $\left(\chi_{\|}\right)$to the $c$ axis were taken from the work on GaSe in Ref. 15.

\section{Variation of phonon linewidths under pressure}

Since our pressurization procedures cause negligible pressure gradients, as was discussed in the previous section, the observed variations of the phonon lines are an intrinsic pressure effect and are not to be assigned to stress inhomogeneity within the cell. The FWHM of one-phonon lines are reported in Fig. 4 for the $E_{1 g}$ and $E_{2 g}$ modes and in Fig. 5 for the $A_{1 g}$ modes.

As was already mentioned, $A_{1 g}$ modes are the most intense in the spectrum, and therefore it was possible to measure their FWHM up to $170 \mathrm{kbar}$, instead of $70 \mathrm{kbars}$ or less for the $E_{1 g}$ and $E_{2 g}$ modes.

\section{DISCUSSION}

\section{A. One-phonon modes}

At low pressure, the values of the pressure coefficients of the Raman-active phonons are similar to previously reported results. ${ }^{5,16}$ The low-frequency $E_{2 g}$ mode exhibits a relative pressure coefficient which is about 10 times higher than that of the four high-frequency modes. This is expected since the low-frequency $E_{2 g}$ mode is an interlayer shear vibration, whereas the upper four mainly involve intralayer restoring forces. It may be noted here that the $E_{2 g}$ mode, originally at 23 $\mathrm{cm}^{-1}$, could be observed up to $76 \mathrm{~cm}^{-1}$ around 150 kbars. This corresponds to an increase of a factor 10 in the interlayer interaction (equivalent spring rigidity). In our lattice dynamical model, ${ }^{17}$ using axially symmetric forces and a rigid ion model, we found that intralayer springs at room pressure were one order of magnitude larger than interlayer restoring forces. Thus, an increase of a factor 10 in the latter brings them into the range of intralayer forces. Above $150 \mathrm{kbars}$, or so, GaS is, in that respect, a quasi-three-dimensional crystal. This view is consistent with the fact that, at the highest pressures, the pressure coefficients of all modes, except the lower $E_{1_{g}}$, become similar, in sharp contrast with the behavior at low pressure. This remark will be used 


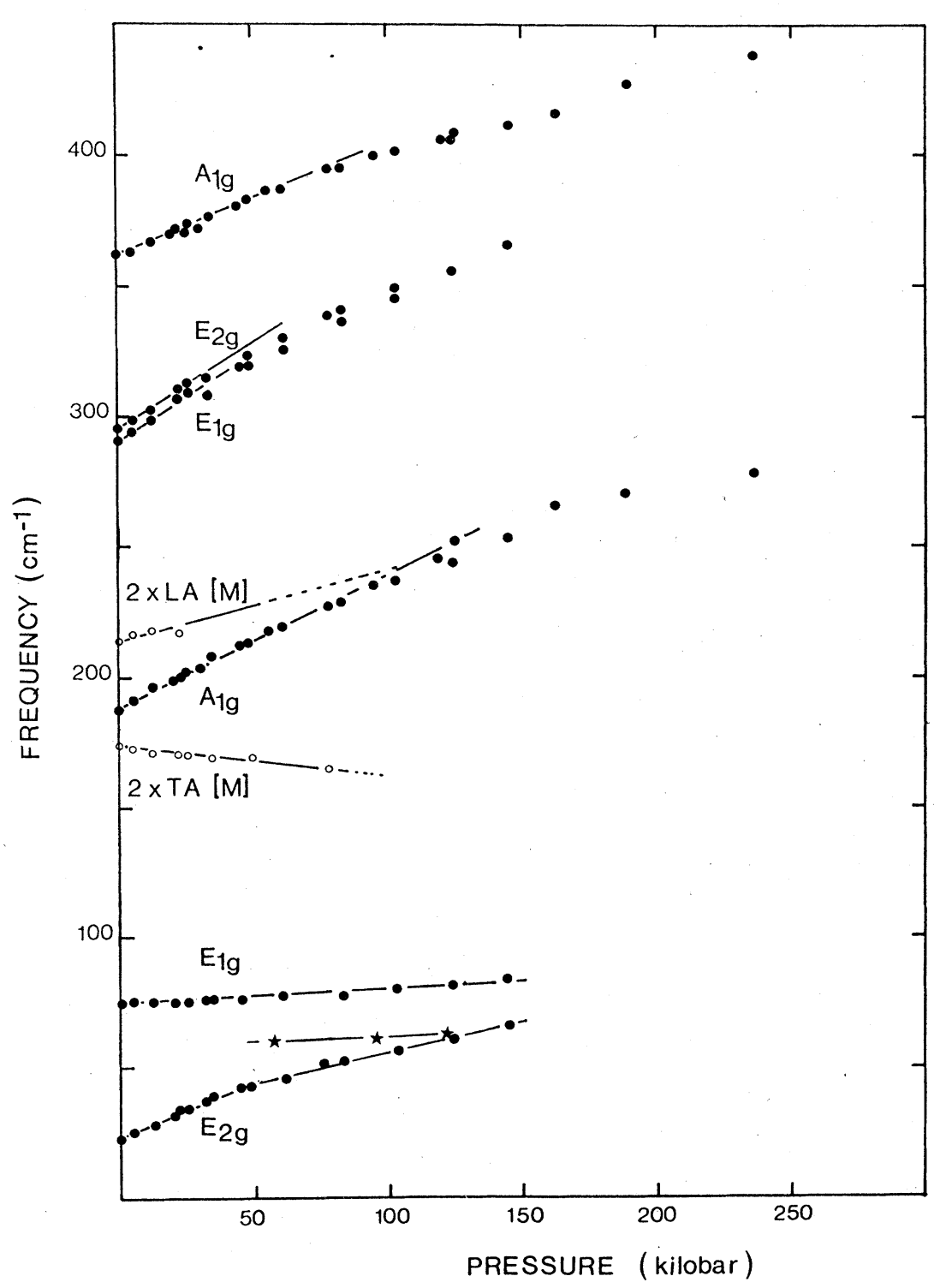

FIG. 3. Trajectories of the Raman-allowed modes versus pressure. The one-phonon lines are indicated by points, edge-of-the-zone overtones by circles, and the unidentified extra phonon (see text) by stars.

to propose an explanation for the "singular" behavior of the $74-\mathrm{cm}^{-1} E_{1 \mathrm{~g}}$ mode.

The pressure behavior of normal modes in lowdimensionality crystals has been discussed in a general fashion by Zallen. ${ }^{3}$ In his approach, molecules are units with strong first-order bonding; layers in $2 \mathrm{D}$ crystals, chains in $1 \mathrm{D}$ crystals, or molecules in purely molecular crystals. Then only intermolecular modes have Grüneisen parameters (see Table I) of the order of unity which are similar to those of isotropic $3 \mathrm{D}$ crystals since intermolecular distances take the bulk of the volume decrease under pressure. On the contrary, intramolecular modes are less susceptible to the effect of pressure and their Grüneisen parameter, which is proportional to $(1 / \nu)(d \nu / d P)$, decreases as $\nu^{-2}$. This phenomenological scaling law was applied to a variety of molecular crystals ${ }^{3,4}$ and to layered $\mathrm{As}_{2} \mathrm{~S}_{3}$ (Refs. 2 and 3).

In Fig. 6, we have plotted the relative pressure variation of the six observed modes as a function of frequency at zero pressure. For comparison, a straight line representing an inverse quadratic dependence of $(1 / \nu)(d \nu / d P)$ (or $\left.\gamma_{i}\right)$ versus frequency was fitted with the four high-frequency intralayer $^{17}$ modes. The low-frequency $E_{1 g}$ mode clearly stands out since its Grüneisen parameter is about one order of magnitude too low to fit even 


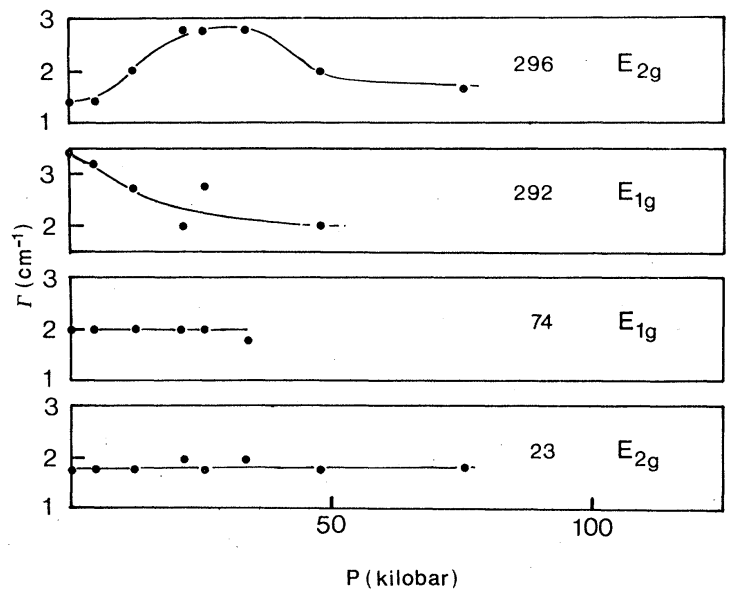

FIG. 4. Full width at half maximum (FWHM) of the $E_{1 g}$ and $E_{2 g}$ phonons versus pressure.

with the intramolecular $\gamma_{i} \sim \nu_{i}^{-2}$ law. This mode is a half-layer shear mode, involving at room pressure mainly Ga-Ga restoring forces (Fig. 7). In view of the nature of this mode, a tentative explanation can be given along the following argument: Since this mode does not change the Ga-S distance within the same half-layer, we will view this vibration as a transverse acoustical (TA) mode of GaS molecules, on a chain along the $c$ axis. At room pressure, those molecules are alternatively linked by strong Ga-Ga bonds and weak interlayer springs. The latter are very strongly affected by pressure, as shown by the high initial pressure dependence of the interlayer shear $E_{2 g}$ mode. At high pressure, both kinds of interactions are expected to become more and more similar. Let us, for argument's sake, consider them as equal (high-pressure limit). In this case, the displacement pattern in Fig. 7 is that of a zone boundary TA phonon propagating along the $c$ axis. Its sine dispersion curve is schematized as a full line in Fig. 8 for a system with one half-layer (one GaS molecule) periodicity. Folding of this Brillouin zone represents the introduction of different inter and intralayer bonds: We have now one-layer periodicity ( $2 \mathrm{GaS}$ molecules per unit cell). The

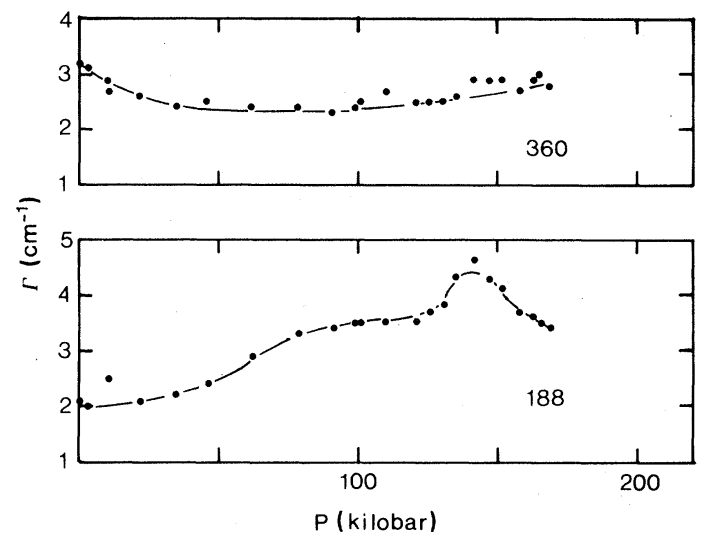

FIG. 5. FWHM of the $A_{1 g}$ phonons versus pressure.

TA mode at the edge of the zone is folded back to the center (upper dashed line) but retains the same displacement pattern as the original $E_{1 g}$ mode in the actual GaS crystal.

In this intermediate step [BZ edge at $(0,0,1) \pi /$ $2 c$, the edge-of-the-zone vibration has the character of a rigid-layer mode in the GaS crystal (two GaS molecules shifting in phase in the same layer). A second folding of the $\mathrm{BZ}$ introduces the pairing of two layers which brings us to the actual $\mathrm{GaS}$ unit cell (4 GaS molecules) and brings the rigid-layer mode to the center of the zone. It should be borne in mind that all this argument is applicable in the high-pressure limit. If it is valid, we should expect the frequency of the rigid half-layer mode $\left(E_{1 g}\right)$ and the rigid-layer mode $\left(E_{2 g}\right)$ to be in the ratio of $\sqrt{2}$ to 1 . Indeed, taking the observed values at $150 \mathrm{kbars}$, we find that $E_{2 g} / E_{1 g}=\frac{66}{84} \simeq 0.79$ instead of the expected $\sqrt{2} / 2$ $\simeq 0.71$, which is surprisingly close, in view of the approximations.

The conclusion of this estimate is as follows: The bond-stretching pattern of the center of zone$E_{1 g}$ mode is analogous to that of an edge-of-thezone TA mode in a linear chain and thus also in a three-dimensional crystal. This has been shown by using the pressure dependence of the $E_{1 g}$ and $E_{2 g}$ modes, but this admixture of TA-like character is true at all pressures for the $E_{1_{g}}$ mode

TABLE I. Pressure coefficient and approximate Grüneisen parameter [calculated with isothermal compressibility of GaSe (Ref. 15)] of the Raman-allowed phonons of GaS.

\begin{tabular}{cllccccccc}
\hline \hline & \multicolumn{4}{c}{ One-phonon modes } & \multicolumn{3}{c}{ Two-phonon modes } \\
\hline Room-pressure wave number $\left(\mathrm{cm}^{-1}\right)$ & 23 & 74 & 188 & 292 & 296 & 360 & 175 & 215 \\
Symmetry or nature assignment & $E_{2 g}$ & $E_{1 g}$ & $A_{1 g}$ & $E_{1 g}$ & $E_{2 g}$ & $A_{1 g}$ & $2 \mathrm{TA}(M)$ & $2 \mathrm{LA}(M)$ \\
Pressure coefficient & 22 & 1 & 2.5 & 2.2 & 2.2 & 1.5 & -1.0 & 1.2 \\
$\quad(1 / \nu)(d \nu / d P)$ in $10^{-6}$ bar $^{-1}$ & 16.9 & 0.8 & 1.9 & 1.7 & 1.7 & 1.2 & -0.8 & 0.9 \\
Approximate Grüneisen parameter & & & & & & & & \\
\end{tabular}




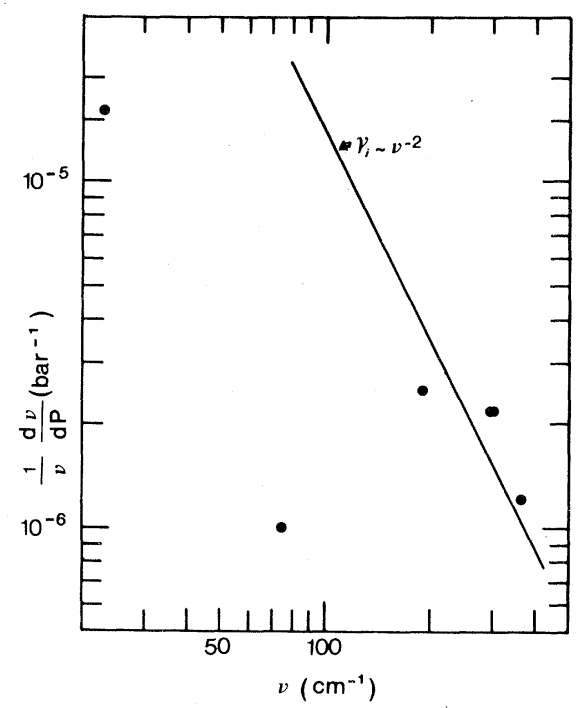

FIG. 6. Pressure coefficient of the one-phonon lines versus frequency. The continuous line represents $\gamma_{i}$ $\sim \nu_{i}^{-2}$.

which is not very much affected by pressure, that is, by increasing interlayer interaction.

We will now proceed to link the singular pressure coefficient of the $E_{1 g}$ mode to this TA shear distortion contribution: Edge-of-the-zone TA modes in 3D crystals are known to exhibit negative Grüneisen parameters. This is because shear distortions destabilize the ionic system since nearest-neighbor distances increase to second order in the strain. The electronic contribution is responsible for the stability of the structure. When pressure is applied, the electronic distribution tends to be spread out in most crystals, and this delocalization of the charges decreases the stabilizing electronic contribution, softens the total restoring spring, and results in a negative pressure coefficient. This explanation is entirely applicable to the rigid half-layer shear mode $\left(E_{1 g}\right)$ in GaS: Here, an important electronic contribution to the relevant bond (Ga$\mathrm{Ga}$ ) is due to the overlapping of the $d$ orbitals on gallium. It has been shown that, for an isolated layer, these orbitals point inside the layer and are entirely localized within the layer. When interlayer interaction is switched on, the $d$ orbitals partially point outside. ${ }^{18}$ High pressure, which increases interlayer ${ }^{18}$ interaction in this class of crystals, ${ }^{19}$ also increases the delocalization of those electrons over the interlayer space and thus brings in a negative contribution to the restoring forces. In the case of $E_{1 g}$, this contribution is not sufficient, as in the case of TA modes in 3-dimensionally bonded crystals, to overcome the increase due to other contributions;
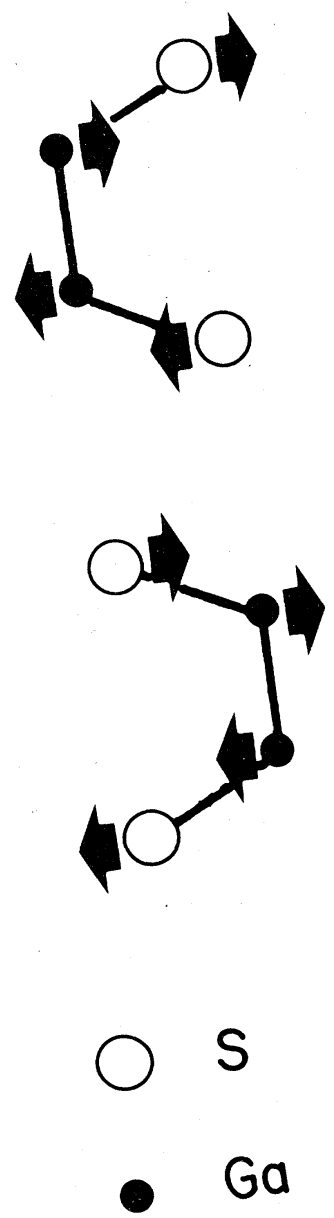

FIG. 7. Displacement pattern of the $E_{1 g}$ half-layer shear mode. One $D_{6 h}$ unit cell of $\beta \mathrm{GaS}$ is represented. Arrows are in the layer plane and represent the displacement pattern of individual atoms for the $74 \mathrm{~cm}^{-1}$ $E_{1 g}$ vibration.

instead of leading to an absolutely negative coefficient, it results in a weakly positive value.

In summary, we propose to explain the singular value of $(1 / \nu)(d \nu / d P)$ for the $74-\mathrm{cm}^{-1} E_{1 g}$ mode as the result of two contributions:

a positive one coming from the decrease with pressure of the intralayer distances;

a negative one coming from the shear motion, and the TA-like character in this mode. The total may be either weakly positive, zero, or negative, but in any case, in algebraic value much less than for other intralayer modes.

A final remark on this type of vibration: This discussion is clearly valid for all crystals with mirror-plane symmetry in the layer. For example, the same "anomalous" pressure coefficient exists in the $E^{\prime \prime}$ mode of GaSe which is also rigid half-layer shear. It is not observed in ${ }^{\circ} \mathrm{As}_{2} \mathrm{~S}_{3}$ since such a mode cannot exist, due to the 


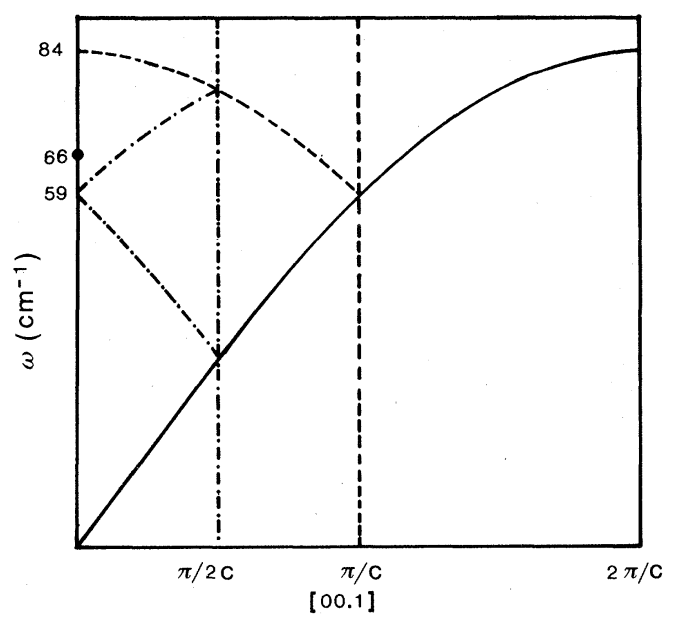

FIG. 8. Example of folding of the Brillouin zone in the $[0,0,1]$ direction. $84 \mathrm{~cm}^{-1}$ is the actual value of the $E_{1 g}$ mode at $150 \mathrm{kbars} .59 \mathrm{~cm}^{-1}$ is the frequency of the "folded" rigid-layer mode at this pressure, and the full circle at $66 \mathrm{~cm}^{-1}$ is the actual frequency of the mode.

layer symmetry.

Apart from the Grüneisen parameter for the rigid half-layer shear mode $E_{1 g}$ a second point may be noted with reference to Fig. 6 (Refs. 3 and 4) and the work on molecular crystals and $\mathrm{As}_{2} \mathrm{~S}_{3}$; that is, the value of the Grüneisen parameter for the rigid-layer mode $(\mathrm{rlm}) E_{2 g}$ at $23 \mathrm{~cm}^{-1}$ at room pressure. Its value, computed as described in the second paragraph of Sec. II is about $\gamma \simeq 17$ which is one order of magnitude too high to compare with the values commonly found in the molecular crystals, three-dimensionally bounded crystals, or $\mathrm{As}_{2} \mathrm{~S}_{3}$ where it is of the order of unity. This cannot be ascribed to the fact that we used the values of compressibility for GaSe which are surely somewhat different from those, unknown yet, for $\mathrm{GaS}$; in the few instances ${ }^{20}$ when those could be compared in isostructural and isoelectronic layer sequences, they were found to be similar. In any case, if one compares exactly with GaSe which has a similar structure and where $(1 / \nu)(d \nu / d P)$ is known from previous work ${ }^{6,16}$ for the $\mathrm{rlm}$, one also finds $\gamma\left(E^{\prime \prime}\right) \simeq 15$, using the compressibility of Ref. 15.

The conclusion is that the use of an isotropic scalar compressibility to derive Grüneisen parameter of interlayer or interchain vibrations in highly anisotropic (1D or 2D) structures may lead to unreliable values even for order-of-magnitude comparison with three-dimensionally bonded structures. On the contrary, ${ }^{8,21}$ this is apparently legitimate in the case of intermolecular vibrations in molecular crystals which are a good three-dimensional analog of 3D crystals where atoms are replaced by individual molecules.
B. Two-phonon modes

Dispersion curves of acoustical branches in GaS have been measured by inelastic neutron scattering ${ }^{17,21}$ in the $A[0,0,1], \Sigma[1,0,0]$, and $T[1,1,0]$ directions, but in the latter not up to the edge-of-the zone ( $K$ point). At the $M$ point ( $\Sigma$ direction), the out-of-plane TA phonon has a frequency of $88 \pm 2 \mathrm{~cm}^{-1}$ and the LA of $104 \pm 3 \mathrm{~cm}^{-1}$. The weak Raman peaks observed at $175 \pm 1 \mathrm{~cm}^{-1}$ and $215 \pm 1 \mathrm{~cm}^{-1}$ fit well with the values expected from neutron data for $2 \mathrm{TA}(M)=176 \pm 4 \mathrm{~cm}^{-1}$ and $2 \mathrm{LA}(M)=208 \pm 6 \mathrm{~cm}^{-1}$. The observed overtones might also originate, in part or in totality, from densities of states at the $K$ point where the frequencies should be somewhat different from the $M$ point.

The negative value of the pressure coefficient of the TA mode is similar to those found in $3 \mathrm{D}$ network crystals such as $\mathrm{Si}, \mathrm{Ge}$, III-V, and II-VI compounds. ${ }^{8}$ It is the first time this behavior is observed outside crystals with three-dimensional molecular units. This tends to confirm that even outside this class of lattices, the negative pressure coefficient of zone-edge phonons is a general property, related to the distortion involved in this type of shear vibration.

Two-phonon densities of states also have resonant interference with one-phonon peaks when their energies meet under pressure. This is seen as an increase in the linewidths of the latter. In Fig. 3 , the $A_{1 \mathrm{~g}}$ mode, $188 \mathrm{~cm}^{-1}$ at room pressure is expected to cross the 2-LA line around $100 \mathrm{kbar}$, because of the difference in their pressure coefficient. This results (Fig. 5) in a broadening, in that region of the $A_{1 g}$ linewidth. Above 140 kbars, the two modes split apart and the FWHM decreases again. The general shape of the $\Gamma(P)$ curve for the low-frequency $A_{1 g}$ mode may be directly related to the edge-of-the-zone density of states (versus frequency). Comparison between the FWHM of the two $A_{1 g}$ modes gives proof that no "spurious" broadening due to stress inhomogeneity disturbs the width measurement: The high-frequency mode broadens by no more than $0.5 \mathrm{~cm}^{-1}$, whereas the low-frequency mode broadening is more than $2.5 \mathrm{~cm}^{-1}$. If stress inhomogeneity were responsible for this, the same behavior should be observed on both vibrations, which is not the case.

The other $A_{1 g}$ mode, $360 \mathrm{~cm}^{-1}$ at room pressure, experiences an immediate decrease of its linewidth which levels off above $20 \mathrm{kbars}$. Comparison with known dispersion curves ${ }^{17,21}$ shows that it may have been degenerate with combinations of edge-of-the-zone $B_{1}$ phonons which separate in energy with increasing pressure. It 
should be noted, at this stage, that the relative intensity of the two $A_{1 g}$ modes changes by a factor of 10 between 0 and $170 \mathrm{kbars}$, the high-frequency mode $\left(360 \mathrm{~cm}^{-1}\right.$ at room pressure) becoming dominant at high pressure.

Large variations of the Raman tensor under stress are a characteristic feature of crystals with low-dimensionality networks, layer structures in particular: It can be traced back directly to the existence of weak (second-order) interlayer bonds. Although those bonds make up a small fraction of the restoring forces for intralayer modes to first order, they are responsible for most of their Raman efficiency since they originate from highly polarizable electronic distributions. This is the reason for the remarkably large Raman efficiency of layer networks and also for its strong variations under stress ${ }^{2,5}$ : In the present case we could identify variations of interlayer forces of 1 to 10 . It is therefore natural to expect variations of polarizability of the same order of magnitude, and to observe correspondent variations of the polarizability derivatives (Raman efficiency). Ab initio calculations of Raman cross sections have been performed as yet only for the simplest cases (e.g., silicon). In the future, when they can be applied to layer crystals, this class of networks will be a very sensitive test of the calculations, because of the large variation of Raman efficiencies which they exhibit in the same crystal structure.

The doublet of $E_{1 g}-E_{2 g}$ frequencies at 292 and $296 \mathrm{~cm}^{-1}$ shows interesting features (Fig. 4). The $E_{1 g}$ linewidth decreases at low pressures and then remains constant. On the contrary the $E_{2 g}$ linewidth exhibits a maximum $30 \mathrm{kbars}$ higher and then drops back to its initial linewidth. The simplest explanation for this behavior is the presence in this energy range of a combined density of states which shifts up in pressure by $(1 / \nu)(d \nu /$ $d P$ ) of the order of $3 \times 10^{-6}$ bar, ${ }^{-1}$ from about 293 $\mathrm{cm}^{-1}$ at room pressure. This interpretation is not unique but it points to the existence in this region of at least one joint-density-of-states singularity which was not otherwise observable, being either Raman forbidden or simply too weak. This type of behavior which has been noted before ${ }^{1}$ actually provides a new method in phonon spectroscopy ${ }^{22}$ by allowing direct observation of phonon densities of states which are Raman and infrared inactive.

\section{Optical behavior and phase transition}

The behavior of optical properties in samples which have been subjected to pressures in the 200-kbar range, as described in Sec. II cer- tainly suggests the occurrence of some kind of structural phase transition. Although this is not the main point of this paper, it is still possible to propose a preliminary discussion of our data:

(i) Evidence for a structural change is clear in Fig. 2 from the position and slope of the absorption edges at $30 \mathrm{kbars}$ before and after taking the sample to over $180 \mathrm{kbars}$.

(ii) The occurrence of an extra Raman-active mode around $60 \mathrm{~cm}^{-1}$ (stars in Fig. 3) after pressurizing above $180 \mathrm{kbars}$, is also an indication of a modification in the bond pattern in GaS.

One possibility for the sudden blackening of the crystal above $180 \mathrm{kbars}$ might have been a chemical decomposition process, analogous to that which has been proposed for GaP at 240 kbars. ${ }^{10}$ This can be ruled out for two reasons:

(i) If this were the case, e.g., precipitation of $\mathrm{Ga}$ in a $\mathrm{Ga}_{2} \mathrm{~S}_{3}$ matrix, one would expect the crystal to remain opaque down to room pressure, as happens in GaP (Ref. 10) or CdS (Ref. 23).

(ii) Analysis of the absorption coefficient of GaS after the phase transition, back at $30 \mathrm{kbars}$ (Fig. 9) shows a remarkably regular quadratic absorp-

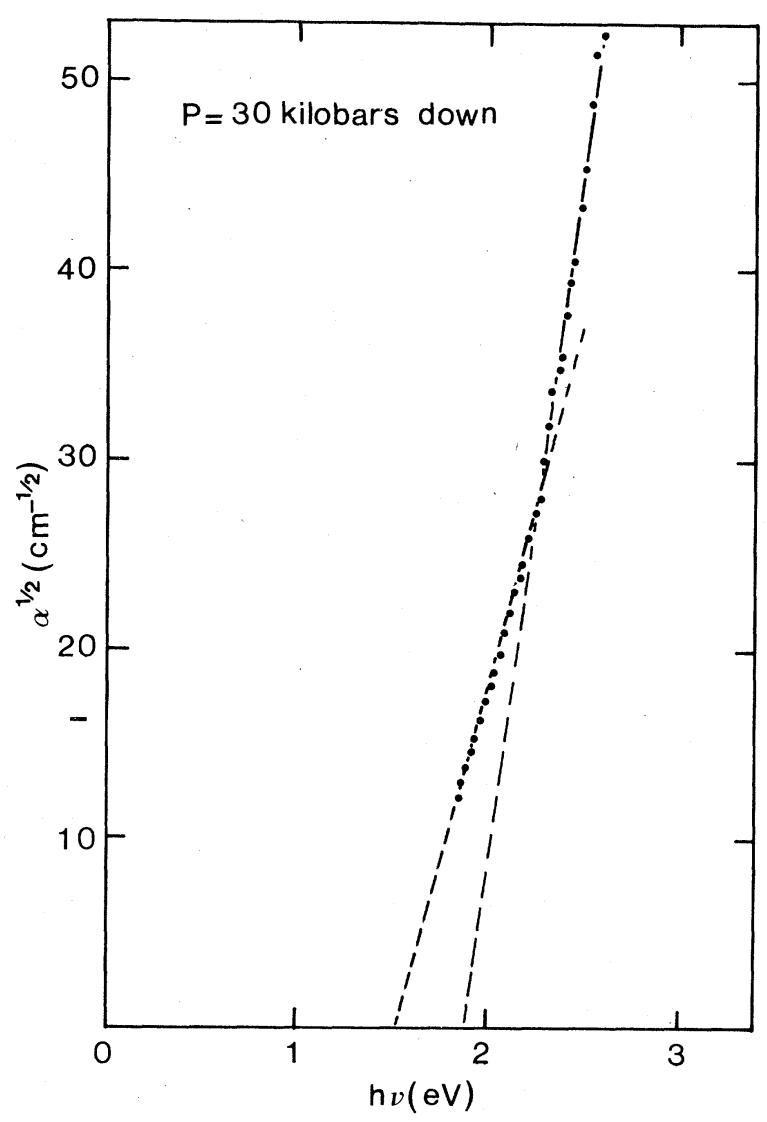

FIG. 9. Square root of the absorption coefficient versus photon energy from curve 2 of Fig. 2. 
tion law. Figure 9 is just curve 2 of Fig. 2 plotted along a $a \sim(E-E g)^{2}$ dependence. In the case where only diffusion on precipitate centers or clusters was responsible for the extra-low-energy absorption, one would expect the $\alpha^{1 / 2} \sim h \nu$ plot to extrapolate to zero (very low) photoenergy $h \nu$ to follow classical optical diffusion laws. This is not the case.

This behavior would be consistent with the sample being a bona fide crystalline semiconductor with two indirect energy gaps at 1.90 and 1.50 $\mathrm{eV}$, at $30 \mathrm{kbars}$, that is much lower than in $\beta-\mathrm{GaS}$ at $30 \mathrm{kbars}$ which is at $2.30 \mathrm{eV} .^{6,13}$ It is tempting to relate this decrease of the energy gap to a structural modification where interlayer bonds would be increased so much that they would compare with intralayer bonds, after a drastic decrease of interlayer spacing.

In this scheme, the individual layers, or at least the hexagonal half-layers, would undergo little perturbation, whereas the interlayer forces would be of the same order of magnitude as the intralayer $\mathrm{Ga}-\mathrm{Ga}$ bonds which join each halflayer. Then the crystal would be much closer to three-dimensional first-order binding than in the usual $\beta$-type layer modification. The experimental facts which support this proposal can be summed up as follows:

(1) The decrease of the indirect optical edge in the high-pressure modification. In this class of semiconductors, its decrease under pressure has been observed ${ }^{5,6}$ from the increase of interlayer interaction, due to pressure, of the individual layers. Thus, the large decrease of the gap is consistent with the hexagonal layers getting packed closer together.

(2) At least, one extra phonon was observed during the decrease of pressure (stars in Fig. 3). This extra line will not be attributed until the high-pressure structure is known.

\section{CONCLUSION}

The main points that have been made in this study can be summed up as follows: The rapidpressurization method which we have devised does indeed allow extension of measurements under definite (homogeneous) stress well above 100 kbars. Although, at room temperature, there is no possibility of extending the range of strictly hydrostatic measurements above 115 kbars (Ref. 24) $\left({ }^{4} \mathrm{He}\right.$, precise pressure data can be obtained in glassy mixtures by our method above 300 kbars. $^{10}$. The application to interference phonon spectroscopy under pressure is demonstrated here on GaS. Systematic exploration of crossing and uncrossing of phonon frequencies may prove as powerful a tool for lattice vibrations as was the same method when applied to electronic states of semiconductors ( $\mathrm{GaSb}, \mathrm{GaAs}$, etc.,...) by $\mathrm{Paul}^{25}$ and co-authors 15 years ago. This is especially clear when it allows, as in the case of $\mathrm{GaS}$, observation of states which are Raman and infrared inactive.

The destabilizing effect of pressure on certain bonds through the variation of the electronic contribution is shown to be present in this class of crystals in the same fashion as in 3D networks, and will account for the negative pressure coefficient of the edge-of-the-zone TA and the small positive coefficient of the intralayer shear $E_{1 \mathrm{~g}}$. In the case of $\mathrm{GaS}$, direct use of an isotropic Grüneisen coefficient to characterize modes does not seem as justified as in other low-dimensionality crystals.

Finally, the evidence we present on the highpressure phase transition, incomplete as it may be, still points to a new modification with $3 \mathrm{D}$ bonding characteristics. It should be borne in mind that there are very few examples of direct transitions for crystal networks from 2D to 3D. Graphite to diamond, for example, takes place at $1480{ }^{\circ} \mathrm{C}$ and $55 \mathrm{kbars}$ through a nickel-rich intermediate phase which thus involves complete reconstruction of the network. This is not the case here, since the transition we report on apparently does not involve complete reconstruction of the individual layers. Structural information under high pressure is nevertheless necessary to draw final conclusions in regard to this point.
${ }^{1}$ B. A. Weinstein and G. J. Piermarini, Phys. Rev. B 4, 1172 (1975).

${ }^{2} \mathrm{~J}$. M. Besson, J. Cernogora, and R. Zallen, to be published in Phys. Rev. B.

${ }^{3}$ R. Zallen, Phys. Rev. B 9, 4485 (1974).

${ }^{4}$ R. Zallen and M. L. Slade, Phys. Rev. B 18, 5775 (1978).

${ }^{5}$ A. Polian, K. Kunc, and A. Kuhn, in the Proceedings of the 13th International Conference on the Physics of
Semiconductors, Roma, 1976, edited by S. G. Fumi (Istituto di Fisica, Rome, 1977), p. 392.

${ }^{6} J$. M. Besson, R. Le Toullec, J. P. Pinceaux, A. Chevy, and H. Fair, High Temp. High Pressures 7, 720 (1975).

${ }^{7}$ J. M. Besson and J. P. Pinceaux, Rev. Sci. Instrum. 50, 79 (1979).

${ }^{8} \mathrm{An}$ extensive bibliography on the subject is given in G. Martinez, Handbook on Semiconductors, edited by 
M. Balkanski (North-Holland, Amsterdam), Vol. 2, p. 181.

${ }^{9}$ A. Polian, J. M. Besson, and J. C. Chervin, VIII AIRAPT International Conference on High Pressure, Le Creusot, 1979, edited by B. Vodar (Pergamon, New York, 1980), p. 517.

${ }^{10}$ J. P. Pinceaux, J. M. Besson, G. Weil, and A. Rimsky, Ref. 9.

${ }^{11}$ G. J. Piermarini, S. Block, and J. D. Barnett, J. Appl. Phys. 44, 5377 (1973).

${ }^{12}$ G. J. Piermarini, R.A. Forman, and S. Block, Rev. Sci. Instrum. 49, 1061 (1978).

${ }^{13} \mathrm{M}$. Mejatty, A. Segura, R. Le Toullec, J. M. Besson, A. Chevy, and H. Fair, J. Phys. Chem. Solids 39, 25 (1978).

${ }^{14}$ A. Polian, J. C. Chervin, and J. M. Besson, Rap. Sci. Lab. Phys. Solids 14, 49 (1978).

${ }^{15}$ N. G. Aliev, I. G. Kerimov, M. M. Kurbanov, and T. A. Mamedov, Fiz. Tverd. Tela. (Leningrad) 14 , 1522 (1972) [Sov. Phys. Solid State 14, 1304 (1972)]. ${ }^{16}$ R. N. Tyte, W. Richter, and W. Rautenberg, Verh.
Dtsch. Phys. Ges. (VI) 10, 403 (1975).

${ }^{17}$ A. Polian, K. Kunc, A. Kuhn, and B. Dorner, in the Proceedings of the 14th I. C. P. S., Edinburgh, 1978 (Institute of Physics, London, 1979), p. 907.

${ }^{18}$ A. Bourdon, A. Chevy, and J. M. Besson, Ref. 17, p. 1371.

${ }^{19}$ J. M. Besson, K. P. Jain, and A. Kuhn, Phys. Rev. Lett. 32,936 (1974).

${ }^{20}$ A. J. Grant, J. A. Wilson, and A. D. Yoffe, Philos. Mag. 25, 625 (1972).

${ }^{21}$ B. M. Powell, S. Jandl, J. L. Brebner, and F. Levy, J. Phys. C 10, 3039 (1977).

${ }^{22}$ D. Petritis, G. Martinez, C. Levy-Clement, and O. Gorochov (unpublished).

${ }^{23}$ C. G. Homan, Ref. 9.

${ }^{24}$ J. M. Besson, J. P. Maury, and J. P. Pinceaux, J. Phys. Lett. 40, L307 (1979); J. M. Besson and J. P. Pinceaux, Science 206, 1073 (1979).

${ }^{25} \mathrm{~W}$. Paul, Propriétés Physiques des Solides sous Pression, Colloque CNRS, Grenoble, 1969 (Editions du CNRS, Paris, 1970), p. 199. 